\title{
New Chiral Universality Class in a Frustrated Three-Leg Spin Ladder
}

\author{
P. Azaria ${ }^{1}$, P. Lecheminant ${ }^{2}$, and A. A. Nersesyan ${ }^{3,4}$ \\ ${ }^{1}$ Laboratoire de Physique Théorique des Liquides, Université Pierre et Marie Curie, 4 Place Jussieu, 75252 Paris, France \\ ${ }^{2}$ Laboratoire de Physique Théorique et Modélisation, Université de Cergy-Pontoise, Site de Saint Martin, 2 avenue Adolphe \\ Chauvin, 95302 Cergy-Pontoise Cedex, France \\ 3 Physikalisches Institut, Universität Bonn, Nußallee 12, 53115 Bonn, Germany \\ ${ }^{4}$ Institute of Physics, Tamarashvili 6, 38007\%, Tbilisi, Georgia
}

(Received: )

\begin{abstract}
We study a model of three $S=1 / 2$ antiferromagnetic Heisenberg spin chains weakly coupled by on-rung and plaquette-diagonal interchain interactions. It is shown that the model exhibits a critical phase with central charge $C=2$ and belongs to the class of "chirally stabilized" liquids recently introduced by Andrei, Douglas, and Jerez. By allowing anisotropic interactions in spin space, we find an exact solution at a Toulouse point which captures all universal properties of the model, including the $\mathrm{SU}(2)$ symmetric case. At the new critical point the massless degrees of freedom are described in terms of an effective $S=1 / 2$ Heisenberg spin chain and two critical Ising models. We discuss the spectral properties of the model, compute spin-spin correlation functions and estimate the NMR relaxation rate.
\end{abstract}

PACS No: 75.10.Jm, 75.40.Gb

In close parallel with qualitative difference between the integer-spin and half-integer-spin antiferromagnetic chains, first predicted by Haldane [1], the universal properties of standard spin ladders, i.e. those with the interchain exchange interaction $\left(J_{\perp}\right)$ across the rungs, dramatically depend on the parity of the number of legs [2]. Ladders with even number of legs are disordered spin liquids with a finite gap in the excitation spectrum, while in odd-legged ladders there exists a gapless branch in the spectrum implying that the spin correlations decay algebraically. In the low-energy limit, the critical behavior of the odd-legged ladders is characterized by the central charge $C=1$ corresponding to an effective $S=1 / 2$ Heisenberg spin chain [3]. The interesting question is whether frustration can lead to new types of infrared (IR) behavior not encountered by the above described two "even-odd" scenarios. The existence of real quasi-1D antiferromagnets with a zigzag interchain interaction, such as $\mathrm{Cs}_{2} \mathrm{CuCl}_{4}$ 沟 and $\mathrm{Cu}_{2}\left(\mathrm{C}_{5} \mathrm{H}_{12} \mathrm{~N}_{2}\right) \mathrm{Cl}_{4}$ [河], indicates that this question is not purely academic. The role of frustration has recently been addressed in a twochain model with a weak zigzag interchain coupling. For an isotropic antiferromagnetic interaction the spectrum has an exponentially small gap, and the ground state is spontaneously dimerized [6, [7]. However, an easy plane XXZ-type anisotropy supports a gapless phase characterized by nonzero local spin currents polarized along the anisotropy axis and algebraically decaying incommensurate spin correlations [8].

Recently another type of frustrated two-leg ladders was discussed [9]. In addition to the standard on-rung coupling $J_{\perp}$, the new model also includes an interaction $J_{\times}$ along both diagonals of elementary plaquettes. In this letter, we consider a three-chain generalization of such a model:

$$
\begin{aligned}
& \mathcal{H}=\sum_{n}\left\{J_{\|} \sum_{j=0,1,2} \mathbf{S}_{j, n} \cdot \mathbf{S}_{j, n+1}+J_{\perp} \mathbf{S}_{0, n} \cdot\left(\mathbf{S}_{1, n}+\mathbf{S}_{2, n}\right)\right. \\
& \left.+J_{\times}\left[\left(\mathbf{S}_{1, n}+\mathbf{S}_{2, n}\right) \cdot \mathbf{S}_{0, n+1}+\left(\mathbf{S}_{1, n+1}+\mathbf{S}_{2, n+1}\right) \cdot \mathbf{S}_{0, n}\right]\right\}
\end{aligned}
$$

where frustration introduced by the interaction $J_{\times}$shows up in a highly nontrivial way. We shall show that in a certain range of parameters the model displays a new type of critical behavior in the IR limit, with the central charge $C=2$, rather than $C=1$ as in usual (nonfrustrated) three-leg ladders [10]. This critical behavior is identified as the universality class of "chirally stabilized" fluids, recently introduced by Andrei et al. [11].

We shall assume that $0<J_{\perp}, J_{\times} \ll J_{\|}$. Under this condition a continuum description can be adopted in which the spin densities in each chain are represented as $\mathbf{S}_{j}(x)=\mathbf{J}_{j}(x)+(-1)^{x / a_{0}} \mathbf{n}_{j}(x)$, where $\mathbf{J}_{j}=\mathbf{J}_{j R}+\mathbf{J}_{j L}$ and $\mathbf{n}_{j}$ are, respectively, the smooth and staggered parts of the magnetization. In the continuum limit the Hamiltonian density of the original model (1) takes the form:

$$
\begin{aligned}
\mathcal{H} & =\sum_{j=0,1,2} \frac{2 \pi v_{j}}{3}\left(\mathbf{J}_{j R} \cdot \mathbf{J}_{j R}+\mathbf{J}_{j L} \cdot \mathbf{J}_{j L}\right) \\
& +g_{1} \mathbf{n}_{0} \cdot\left(\mathbf{n}_{1}+\mathbf{n}_{2}\right)+g_{2} \mathbf{J}_{0} \cdot\left(\mathbf{J}_{1}+\mathbf{J}_{2}\right) .
\end{aligned}
$$

Here the first line describes three decoupled chains in terms of three critical SU(2) 1 Wess-Zumino-NovikovWitten (WZNW) models [12], $v_{j} \sim J_{\|} a_{0}$ being the spin velocities. The second line in (2) includes the interchain coupling terms with the constants: $g_{1}=\left(J_{\perp}-\right.$ $\left.2 J_{\times}\right) a_{0}, \quad g_{2}=\left(J_{\perp}+2 J_{\times}\right) a_{0}$. We stress that in the continuum limit there is no marginally relevant twist perturbation, $\mathbf{n}_{i} \cdot \partial_{x} \mathbf{n}_{j}$, which appears in the description of spin ladders with small zigzag interchain coupling [8, 13].

The two interaction terms in (2) are of different nature. The first one is a relevant perturbation with scaling dimension $d=1$. The second term describes an interaction between the total current of the surface chains, 
$\mathbf{I}=\mathbf{J}_{1}+\mathbf{J}_{2}$ and that of the middle chain. This interaction is only marginal and, as long as $g_{1}$ is not too small, can be discarded. As a result, for generic values of $g_{1}$ and $g_{2}$, the low energy physics of our model will be essentially that of the standard three-leg ladder, and frustration will plays no role (except for renormalization of the mass gaps and velocities [14]). The important point, though, is that in contrast with non-frustrated ladders, the two coupling constants $g_{1}, g_{2}$ can vary independently, and there exists a vicinity of the line $J_{\perp}=2 J_{\times}\left(g_{1}=0\right)$ where the lowenergy properties of the model are mainly determined by the current-current interchain interaction. Remarkably enough, exactly at $g_{1}=0$ an anisotropic version of this model is exactly solvable at a Toulouse point. The solution describes a new fixed point with a larger central charge $C=2$ which, as we shall demonstrate, govern the physics for sufficiently small $g_{1}$. Our strategy will be first to present our solution and discuss the physical properties of the system at the special point $J_{\perp}=2 J_{\times}$, and then to explore its neighborhood for $g_{1}$ small.

Toulouse point solution. We start by neglecting the interaction between currents of the same chirality. Such terms lead to renormalization of the velocities of the excitations which can be effectively taken into account by allowing the surface chain velocities $\left(v_{1}=v_{2}\right)$ to be different from that of the middle chain $v_{0}$. In the noninteracting case $\left(g_{2}=0\right)$, the central charge of two surface chains is $C=2$. On the other hand, the total current I, being the sum of two $\mathrm{SU}(2)_{1}$ currents, satisfies the $\mathrm{SU}(2)_{2}$ Kac-Moody algebra. Since the central charge of the $\mathrm{SU}(2)_{2}$-symmetric WZNW model is $C=3 / 2$, some degrees of freedom should account for the missing $C=1 / 2$. Those are associated with a discrete $\left(\mathrm{Z}_{2}\right)$ nonmagnetic $1 \leftrightarrow 2$ interchange symmetry, and remain decoupled and critical with the central charge $C=1 / 2$. The simplest way to see this is to exploit the equivalence $\mathrm{SU}(2) \times \mathrm{SU}(2) \approx \mathrm{SO}(4)$ and use the representation of two $\mathrm{SU}(2)_{1}$ currents in terms of a quadruplet of real (Majorana) fermions, $\xi^{0}$ and $\xi^{a}(a=1,2,3)$ [7]: $I_{\alpha}^{a}=-\frac{\mathrm{i}}{2} \epsilon^{a b c} \xi_{\alpha}^{b} \xi_{\alpha}^{c}, \quad\left(J_{\alpha 1}-J_{\alpha 2}\right)^{a}=\mathrm{i} \xi_{\alpha}^{a} \xi_{\alpha}^{0}$, where $\alpha=R, L$. Then one easily finds that the contribution of the massless Majorana fermion $\xi^{0}$ decouples from the rest of the spectrum, and the effective Hamiltonian at the special point $g_{1}=0$ reads:

$$
\mathcal{H}=-\mathrm{i} \frac{v_{1}}{2}\left(\xi_{R}^{0} \partial_{x} \xi_{R}^{0}-\xi_{L}^{0} \partial_{x} \xi_{L}^{0}\right)+\overline{\mathcal{H}}\left[\mathbf{I}, \mathbf{J}_{0}\right] .
$$

All nontrivial physics is incorporated in the current dependent part of the Hamiltonian, $\overline{\mathcal{H}}$, describing marginally coupled $\mathrm{SU}(2)_{2}$ and $\mathrm{SU}(2)_{1}$ WZNW models. Notice that $\overline{\mathcal{H}}$ in turn separates into two commuting and chirally asymmetric parts: $\overline{\mathcal{H}}=\mathcal{H}_{1}+\mathcal{H}_{2},\left(\left[\mathcal{H}_{1}, \mathcal{H}_{2}\right]=0\right)$, where

$$
\mathcal{H}_{1}=\frac{\pi v_{1}}{2} \mathbf{I}_{R} \cdot \mathbf{I}_{R}+\frac{2 \pi v_{0}}{3} \mathbf{J}_{0 L} \cdot \mathbf{J}_{0 L}+g_{2} \mathbf{I}_{R} \cdot \mathbf{J}_{0 L},
$$

with $\mathcal{H}_{2}$ obtained from $\mathcal{H}_{1}$ by inverting chiralities of all the currents. The Hamiltonian (11) resembles the twochannel Kondo model [15]: in the latter case the $\mathrm{SU}(2)_{2}$ current $\mathbf{I}_{R}$ describes two-flavor spin excitations of the conduction electrons, while the $\mathrm{SU}(2)_{1}$ current $\mathbf{J}_{0 L}$ is replaced by the local spin density of the impurity spin $S=1 / 2$. Moreover, one can show that (3) corresponds to the Hamiltonian of the two channel Kondo lattice away from half-filling with a nearest neighbour interaction between the impurity spins.

A simple renormalization group analysis reveals that, at $g_{2}>0$, the interaction is marginally relevant. Usually the development of a strong coupling regime is accompanied by a dynamical mass generation and the loss of conformal invariance at the strong coupling fixed point. This is indeed the case for the model of two marginally coupled $S=1 / 2$ spin chain with zigzag interaction [6, [7]. We shall see, however, that due to chiral asymmetry of $\overline{\mathcal{H}}$ the effective interaction, as in the two-channel Kondo model, flows towards an intermediate fixed point where conformal invariance is recovered with a smaller central charge.

The model (4i) is Bethe-Ansatz solvable 16 (see also [11). Here we present an exact solution for a U(1) version of the model, characterized by anisotropic interaction $\left(g_{2} \rightarrow g_{\|}, g_{\perp}\right)$, which allows us to investigate the spectrum of the model and estimate asymptotics of the correlation functions. Our solution is based on a mapping onto Majorana fermions. Using Abelian bosonization, we shall exploit the existence of a Toulouse-like point where the fermions are free 15]. We start with Abelian bosonization of the $\mathrm{SU}(2)_{1}$ current $\mathbf{J}_{0}$. Introducing a massless bosonic field $\varphi$, we have (see, for instance, Appendix A of Ref. [14]): $J_{0 R, L}^{z}=\frac{1}{\sqrt{2 \pi}} \partial_{x} \varphi_{R, L}$, $J_{0 R, L}^{+}=\frac{1}{2 \pi a_{0}} \mathrm{e}^{\mp \mathrm{i} \sqrt{8 \pi} \varphi_{R, L}}$. On the other hand, combining two Majorana fields, $\xi^{1}$ and $\xi^{2}$ to form a Dirac fermion, $\left(\xi^{2}+i \xi^{1}\right) / \sqrt{2}$, and then bosonizing it, we can express the $\mathrm{SU}(2)_{2}$ current $\mathbf{I}$ in terms of a bosonic field $\Phi$ and the Majorana fermion $\xi^{3}: I_{R, L}^{z}=\frac{1}{\sqrt{\pi}} \partial_{x} \Phi_{R, L}$, $I_{R, L}^{+}=\frac{\mathrm{i}}{\sqrt{\pi a_{0}}} \xi_{R, L}^{3} \kappa \mathrm{e}^{\mp \mathrm{i} \sqrt{4 \pi} \Phi_{R, L}}$. An additional fermionic zero-mode operator $\kappa$ has been introduced to ensure the correct commutation relations. Then the Hamiltonian $\overline{\mathcal{H}}$ can be written in the following bosonized form:

$$
\begin{aligned}
\overline{\mathcal{H}} & =v_{0}\left[\left(\partial_{x} \varphi_{R}\right)^{2}+\left(\partial_{x} \varphi_{L}\right)^{2}\right]+v_{1}\left[\left(\partial_{x} \Phi_{R}\right)^{2}+\left(\partial_{x} \Phi_{L}\right)^{2}\right] \\
& -\mathrm{i} \frac{v_{1}}{2}\left(\xi_{R}^{3} \partial_{x} \xi_{R}^{3}-\xi_{L}^{3} \partial_{x} \xi_{L}^{3}\right) \\
& +\frac{g_{\|}}{\sqrt{2} \pi}\left(\partial_{x} \varphi_{L} \partial_{x} \Phi_{R}+\partial_{x} \varphi_{R} \partial_{x} \Phi_{L}\right) \\
& +\frac{\mathrm{i} g_{\perp}}{2\left(\pi a_{0}\right)^{3 / 2}} \xi_{R}^{3} \kappa \cos \left(\sqrt{4 \pi} \Phi_{R}+\sqrt{8 \pi} \varphi_{L}\right) \\
& +\frac{\mathrm{i} g_{\perp}}{2\left(\pi a_{0}\right)^{3 / 2}} \xi_{L}^{3} \kappa \cos \left(\sqrt{4 \pi} \Phi_{L}+\sqrt{8 \pi} \varphi_{R}\right)
\end{aligned}
$$

We now perform a canonical transformation:

$$
\begin{aligned}
& \varphi=\operatorname{ch} \alpha \bar{\Phi}_{2}+\operatorname{sh} \alpha \bar{\Phi}_{1}, \Phi=\operatorname{ch} \alpha \bar{\Phi}_{1}+\operatorname{sh} \alpha \bar{\Phi}_{2} \\
& \vartheta=\operatorname{ch} \alpha \bar{\Theta}_{2}-\operatorname{sh} \alpha \bar{\Theta}_{1}, \Theta=\operatorname{ch} \alpha \bar{\Theta}_{1}-\operatorname{sh} \alpha \bar{\Theta}_{2}
\end{aligned}
$$


where $\vartheta$ and $\Theta$ (respectively $\bar{\Theta}_{1}$ and $\bar{\Theta}_{2}$ ) are the dual fields associated with $\varphi$ and $\Phi$ (respectively $\bar{\Phi}_{1}$ and $\bar{\Phi}_{2}$ ). The cross terms $\partial_{x} \varphi \partial_{x} \Phi$ in Eq. (5) can be eliminated by setting th2 $\alpha=-g_{\|} / \pi \sqrt{2}\left(v_{0}+v_{1}\right)$. One immediately observes that choosing th $\alpha=-\frac{1}{\sqrt{2}}$, which corresponds to a special (though nonuniversal) positive value of $g_{\|}, \quad\left(g_{\|}^{*}=\right.$ $\left.4 \pi\left(v_{0}+v_{1}\right) / 3\right)$, the arguments of the two cosine terms in (5) become those of free fermions, $\cos \left(\sqrt{4 \pi} \bar{\Phi}_{2 L, R}\right)$. Introducing a pair of Majorana fields, $\eta$ and $\zeta$, and using the correspondence: $\psi_{R, L}=\left(\eta_{R, L}+\mathrm{i} \zeta_{R, L}\right) / \sqrt{2}=$ $\left(\kappa / \sqrt{2 \pi a_{0}}\right) \mathrm{e}^{ \pm \mathrm{i} \sqrt{4 \pi} \bar{\Phi}_{2 ; R, L}}$, we finally obtain:

$$
\begin{aligned}
\overline{\mathcal{H}} & =\frac{u_{1}}{2}\left[\left(\partial_{x} \bar{\Phi}_{1}\right)^{2}+\left(\partial_{x} \bar{\Theta}_{1}\right)^{2}\right]-\frac{\mathrm{i} u_{2}}{2}\left[\zeta_{R} \partial_{x} \zeta_{R}-\zeta_{L} \partial_{x} \zeta_{L}\right] \\
& -\frac{\mathrm{i} v_{1}}{2}\left[\xi_{R}^{3} \partial_{x} \xi_{R}^{3}-\xi_{L}^{3} \partial_{x} \xi_{L}^{3}\right]-\frac{\mathrm{i} u_{2}}{2}\left[\eta_{R} \partial_{x} \eta_{R}-\eta_{L} \partial_{x} \eta_{L}\right] \\
& +\mathrm{i} m\left[\xi_{R}^{3} \eta_{L}-\eta_{R} \xi_{L}^{3}\right] .
\end{aligned}
$$

Here $m=g_{\perp} / 2 \pi a_{0}$, and the two renormalized velocities, $u_{1}$ and $u_{2}$, are expressed in terms of the surface and middle chain velocities $v_{1}$ and $v_{0}$ as $u_{1}=\left(2 v_{1}-v_{0}\right) / 3, \quad u_{2}=$ $\left(2 v_{0}-v_{1}\right) / 3$. The first two terms in Eq. (7) describe completely decoupled free massless bosonic and Majorana fields, $\bar{\Phi}_{1}$ and $\zeta$, contributing to criticality with the central charge: $C=1+1 / 2=3 / 2$. Therefore, at the new critical point $\overline{\mathcal{H}}$ effectively represents a gapless $S=1 / 2$ spin chain and a critical Ising model. Coming back to the model (3) and adding the contribution of the singlet Majorana fermion $\xi^{0}$, i.e. one more critical Ising model, the total central charge becomes $C_{T}=2$. The remaining part of the Hamiltonian (7) has a spectral gap $m$ and describes hybridization of the Majorana $\xi^{3}$ and $\eta$ fields with different chiralities. Since the canonical transformation (6) does not mix $\mathcal{H}_{1}$ and $\mathcal{H}_{2}$, the Hamiltonian (7) still decouples into two commuting, chirally asymmetric parts. This reflects the chiral nature of the fixed point.

Physical picture of elementary excitations. There are two different kinds of elementary excitations at the Toulouse point: magnetic excitation described by the field $\bar{\Phi}_{1}$, and nonmagnetic, singlet, excitations associated with the two Majorana fermions $\xi^{0}$ and $\zeta$.

Notice that, due to the mixing of different degrees of freedom reflected in the canonical transformation (6), the "physical" spinons, i.e. those defined as $\sqrt{\pi / 2}$-kinks of the field $\bar{\Phi}_{1}$ describing the effective $S=1 / 2$ spin chain, should not be misleadingly identified as the spinons of the middle chain. To get a better understanding of the structure of spin excitations at the chiral fixed point, let us express the currents $J_{1}^{z}, J_{2}^{z}$ and $J_{0}^{z}$ in terms of the "physical" current $\mathcal{J}^{z}=(1 / \sqrt{2 \pi}) \partial_{x} \bar{\Phi}_{1}$. Using the transformation (6) at the Toulouse point, we find:

$$
\begin{aligned}
J_{1,2 R(L)}^{z} & =\mathcal{J}_{R(L)}^{z}-\frac{\mathrm{i}}{2}\left(\eta_{L(R)} \zeta_{L(R)} \mp \xi_{R(L)}^{3} \xi_{R(L)}^{0}\right), \\
J_{0 R(L)}^{z} & =-\mathcal{J}_{L(R)}^{z}+\mathrm{i} \eta_{R(L)} \zeta_{R(L)} .
\end{aligned}
$$

At energies $|\omega| \gg m$, where all Majorana fields can be considered as massless, Eqs. (8) transform back to the standard definitions of the currents of the three decoupled chains. In this (ultraviolet) limit, one has a picture of three groups of independently propagating spinons. However, in the IR limit $(|\omega| \ll m)$, all Majorana bilinears in (8) are characterized by short-ranged correlations (since the Majorana fermions $\eta$ and $\xi^{3}$ are massive), implying that strongly fluctuating parts of the currents of individual chains are no longer independent; all of them contribute to the formation of a single, physical, current $\mathcal{J}^{z}$. In fact, the physical spinon represents a chirally asymmetric, strongly correlated state of three spinons. Consider, for instance, a right-moving $\sqrt{\pi / 2}$-kink of the field $\bar{\Phi}_{1}$, representing a physical spinon with the spin projection $S^{z}=1 / 2$. According to the exact relation

$$
\mathcal{J}_{R(L)}^{z}=J_{1 R(L)}^{z}+J_{2 R(L)}^{z}+J_{0 L(R)}^{z}
$$

following from (8), such an excitation is a combination of two right-moving spinons of the surface chains, each carrying the spin $S^{z}=1 / 2$, and a left-moving antispinon of the middle chain, with $S^{z}=-1 / 2$. The rigidity of such a state is ensured by a finite mass gap in the $\left(\eta-\xi^{3}\right)$ sector of the model. This peculiar structure of the elementary spin excitations at the chiral fixed point is also reflected by the expression for the velocity $u_{1}$.

Apart from the nontrivial nature of the spinon, the chiral fixed point manifests itself in the existence of two new massless singlet excitations. The Majorana fermion $\xi^{0}$ describes collective excitations of singlet pairs formed on the two surface chains. The nature of the Majorana fermion $\zeta$ is less transparent: it is a highly nonlocal object when expressed in terms of the original spin operators. The best way to understand the role of the singlet excitations is to combine the $\xi^{0}$ and $\zeta$ fields into a single Dirac fermion and then bosonize it. The corresponding massless bosonic field $\tilde{\Phi}_{c}$ resembles the scalar field describing the charge degrees of freedom in the Hubbard model away from half filling in the limit $U=\infty$. We shall hence refer to the gapless Majorana fields as "pseudocharge" excitations which account for the central charge $C=1$. The spin-pseudocharge separation is already manifest at the Toulouse point implying that the leading asymptotics of correlations functions will factorize into the spin and pseudocharge contributions.

Correlation functions. We shall now use the exact solution of the model, found at the Toulouse point, to calculate physical quantities of interest. From Eqs. (8) it follows that, as for a single $S=1 / 2$ Heisenberg spin chain, an external magnetic field $H$ couples only to the massless field $\bar{\Phi}_{1}$. The uniform susceptibility in units of $g \mu_{B}$ is easily found to be $\chi^{-1}=$ $2 \pi u_{1}$. At low temperatures $(T \ll m)$ only the gapless modes $\left(\xi^{0}, \zeta\right.$ and $\left.\bar{\Phi}_{1}\right)$ contribute to the specific heat. Using the general formula $C_{V}=\pi C T / 3 v$, we find: $C_{V}=\frac{\pi T}{3}\left(\frac{1}{2} \cdot \frac{1}{v_{1}}+\frac{1}{2} \cdot \frac{1}{u_{2}}+1 \cdot \frac{1}{u_{1}}\right)$.

We have also computed all spin-spin correlation functions at the Toulouse point (details will be published else- 
where [13]). The leading asymptotics of the uniform part of the correlation functions coincide with that of the effective $S=1 / 2$ spin chain, in agreement with the above discussion. The nature of the chiral fixed point manifests itself in staggered correlations between the spins of the surface chains:

$$
\begin{aligned}
& \left\langle\mathbf{n}_{a}(x, \tau) \mathbf{n}_{b}(0,0)\right\rangle \\
\sim & \delta_{a b} \frac{1}{\left(x^{2}+u_{1}^{2} \tau^{2}\right)^{1 / 2}} \frac{1}{\left(x^{2}+v_{1}^{2} \tau^{2}\right)^{1 / 8}} \frac{1}{\left(x^{2}+u_{2}^{2} \tau^{2}\right)^{1 / 8}}
\end{aligned}
$$

where $a, b=(1,2)$. Notice the factorized contribution of the "pseudocharge" coming with the exponent $1 / 8$. As seen from Eq. (10), there is an intrinsic velocity anisotropy in the pseudocharge sector which might be important when considering the dynamical properties. We emphasize that the exponents in the correlation functions are universal and characterize a new universality class in spin ladders. This is the main result of our work. Another quantity of experimental interest is the NMR relaxation rate $1 / T_{1}$. It is not difficult to show that at low temperature, $1 / T_{1} \sim \sqrt{T}$, in contrast with the Heisenberg chain where $1 / T_{1} \sim$ const.

Stability of the chiral fixed point. With all these results at hand, now we turn to the stability of the chiral fixed point. There are two important questions we shall adress. The first is related to the stability of the Toulouse point with $g_{1}=0 \mathrm{kept}$ fixed, and the second is to examine the behavior of the system when one moves away from the point $J_{\perp}=2 J_{\times}$.

We begin by stressing that our solution is stable provided both $u_{1}$ and $u_{2}$ are positive, the stability condition thus being $1 / 2 \leq v_{0} / v_{1} \leq 2$. So the chiral fixed point is stable in a relatively broad range of velocities. When $g_{\|}$deviates from its Toulouse-point value, the Hamiltonian (7) picks up an extra term $\delta g_{\|}\left(\partial_{x} \bar{\Phi}_{1 L} \zeta_{R} \eta_{R}\right.$ $\left.+\partial_{x} \bar{\Phi}_{1 R} \zeta_{L} \eta_{L}\right)$. Since the field $\eta$ is massive, the expansion in $\delta g_{\|}$does not introduce new infrared singularities, implying that the long-distance behavior of the correlation functions will not be modified except for a velocity and mass renormalization. Therefore, the solution at the Toulouse point captures all universal properties of the chiral fixed point and includes the case $g_{\|}=g_{\perp}$ where the Hamiltonian has the full $\mathrm{SU}(2)$ symmetry.

Now we consider small deviations from the point $g_{1}=$ 0 . At small enough $\left|g_{1}\right|$ it is possible to investigate the effect of the backscattering term $\mathbf{n}_{0} \cdot\left(\mathbf{n}_{1}+\mathbf{n}_{2}\right)$ as a weak perturbation to the chiral fixed point. This perturbation can be shown to be proportional to $\cos \left(\sqrt{\pi} \tilde{\Phi}_{c}\right)$, which is a relevant operator with scaling dimension $1 / 4$. We thus conclude that the backscattering operator opens a gap, $\Delta_{c}$, in the pseudocharge sector but has no effect on the magnetic (spinons) excitations. Standard scaling arguments give an estimate: $\Delta_{c} \sim g_{1}^{4 / 7}$. The chiral fixed point is thus unstable in the far IR limit, and the system will flow to the $C=1$ fixed point of the standard three-leg spin ladder. Of course, the very applicability of the perturbative approach to the chiral fixed point requires that $\Delta_{c} \ll m$, the condition which can always be satisfied for sufficiently small $g_{1}$. Under this condition, there exists an intermediate but still low-energy region $\Delta_{c} \ll E \ll m$ where the $C=2$ behavior caused by frustration is dominant. The physics in this region is universal and cannot be understood without having recourse to the new chiral fixed point. At lower energies, $E \ll \Delta_{c}$, the system will eventually cross over to the conventional critical $C=1$ behavior.

We think that this new fixed point discussed on the present paper might be responsible for new physics in many frustrated ladders such as the three-chain zigzag ladder. This model and the doped case are currently under study. We hope that the chiral-fluid critical state with all its physical properties will be observed in further experiments on spin-ladder systems.

The authors would like to thank D. Allen, N. Andrei, F. Essler, R. Flume, A. Gogolin, C. Lhuillier, A. M. Tsvelik and $\mathrm{Lu} \mathrm{Yu}$ for very helpful conversations. A.N. acknowledges the support from Deutsche Forschungsgemeinschaft. He would also like to thank Vladimir Rittenberg for his kind hospitality and stimulating discussions.

[1] F. D. M. Haldane, Phys. Lett. 85A, 375 (1981).

[2] See, for a review, E. Dagotto and T. M. Rice, Science 271, 618 (1996), and references therein.

[3] D. V. Khveshchenko, Phys. Rev. B 50, 380 (1994).

[4] R. Coldea et al, Phys. Rev. Lett. 79, 151 (1997).

[5] G. Chaboussant et al, Phys. Rev. B 55, 3046 (1997).

[6] S. White and I. Affleck, Phys. Rev. B 54, 9862 (1996).

[7] D. Allen and D. Sénéchal, Phys. Rev. B 55, 299 (1997).

[8] A. A. Nersesyan, A. O. Gogolin, and F. H. L. Essler, to appear in Phys. Rev. Lett., cond-mat/9804005.

[9] Z. Weihong, V. Kotov, and J. Oitmaa, Phys. Rev. B 57, 11439 (1998); X. Wang, cond-mat/9803290.

[10] E. Arrigoni, Phys. Lett. A 215, 91 (1996); K. Totsuka and M. Suzuki, J. Phys. A: Math. Gen. 29, 3559 (1996); D. Schmeltzer and P. Sun, J. Phys.: Condens. Matter 10, 4435 (1998).

[11] N. Andrei, M. Douglas, and A. Jerez, cond-mat/9803134.

[12] I. Affleck, Nucl. Phys. B265, 409 (1986).

[13] P. Azaria, P. Lecheminant, and A. A. Nersesyan, in preparation.

[14] D. G. Shelton, A. A. Nersesyan, and A. M. Tsvelik, Phys. Rev. B 53, 8521 (1996); A. A. Nersesyan and A. M. Tsvelik, Phys. Rev. Lett. 78, 3939 (1997).

[15] V. J. Emery and S. A. Kivelson, Phys. Rev. B 46, 10812 (1992).

[16] A. M. Polyakov and P. B. Wiegmann, Phys. Lett. B 141, 223 (1984). 